Doug Geisler, Eva K. Grebel, and Dante Minniti, eds.

\title{
A Comparative Study of Globular Cluster Systems in UGC 9799 and NGC 1129
}

\author{
Myung Gyoon Lee, Eunhyeuk Kim \\ Astronomy Program, SEES, Seoul National University, Seoul, 151-742, \\ Korea
}

Doug Geisler

Departamento de Física, Grupo de Astronomía, Universidad de Concepción, Casilla 160-C, Concepción, Chile

Terry Bridges

Anglo-Australian Observatory, P.O.Box 296, Epping, NSW 1710, Australia

Keith Ashman

Department of Physic and Astronomy, University of Kansas, Lawrence, $K S$ 66045-2151, USA

Abstract. We present a preliminary analysis of HST-WFPC2 observations of globular cluster systems in the two brightest galaxies, UGC 9799 (cD) and NGC 1129 (non-cD), located in the center of rich clusters.

UGC 9799 is a cD galaxy located in the center of the massive Abell 2052 cluster at $\mathrm{z}=0.035$, and is known to have the largest number of the globular clusters $(\mathrm{N}($ total $) \approx 46,000)$ and the highest specific frequency of globular clusters from the ground-based observations $\left(S_{N}=20 \pm 6\right.$, Harris, Pritchet, \& McClure 1995). On the other hand, NGC 1129 is a giant, but not cD galaxy, located in the center of a rich cluster AWM7 at $\mathrm{z}=0.018$. Its globular cluster system has not yet been studied. The foreground reddenings are known to be $E(V-I)=0.051$ for UGC 9799 and $E(V-I)=0.159$ for NGC 1129. We adopt the redshift distance modulus $(m-M)_{0}=36.0$ for UGC 9799 and $(m-M)_{0}=34.5$ for NGC 1129 based on the Hubble constant of $H_{0}=65 \mathrm{~km} / \mathrm{s} / \mathrm{Mpc}$.

Deep images of these galaxies were obtained using the HST-WFPC2 with $F 555 W(V)$ and $F 814 W(I)$ filters. We have obtained the photometry of the point sources in the images where bright galaxies were subtracted, using the HSTphot package (Dolphin 2000) and the image classification parameters. Our photometry reaches $V \approx 27.2 \mathrm{mag}$ and $I \approx 26.0 \mathrm{mag}$ with $50 \%$ completeness. Figure 1 displays the color-magnitude diagrams of the point sources in UGC 9799 and NGC 1129. In Figure 1 there is seen a vertical structure at $0.8<(V-I)<$ 1.5 extending up to $I \approx 23 \mathrm{mag}$, which represents the globular clusters in these galaxies. Faint blue objects are mostly background compact galaxies.

Figure 2 shows that the $(V-I)_{0}$ color distributions of the bright point sources with $V<26.5$ mag. In Figure 2 the dominant peaks are due to the glob- 


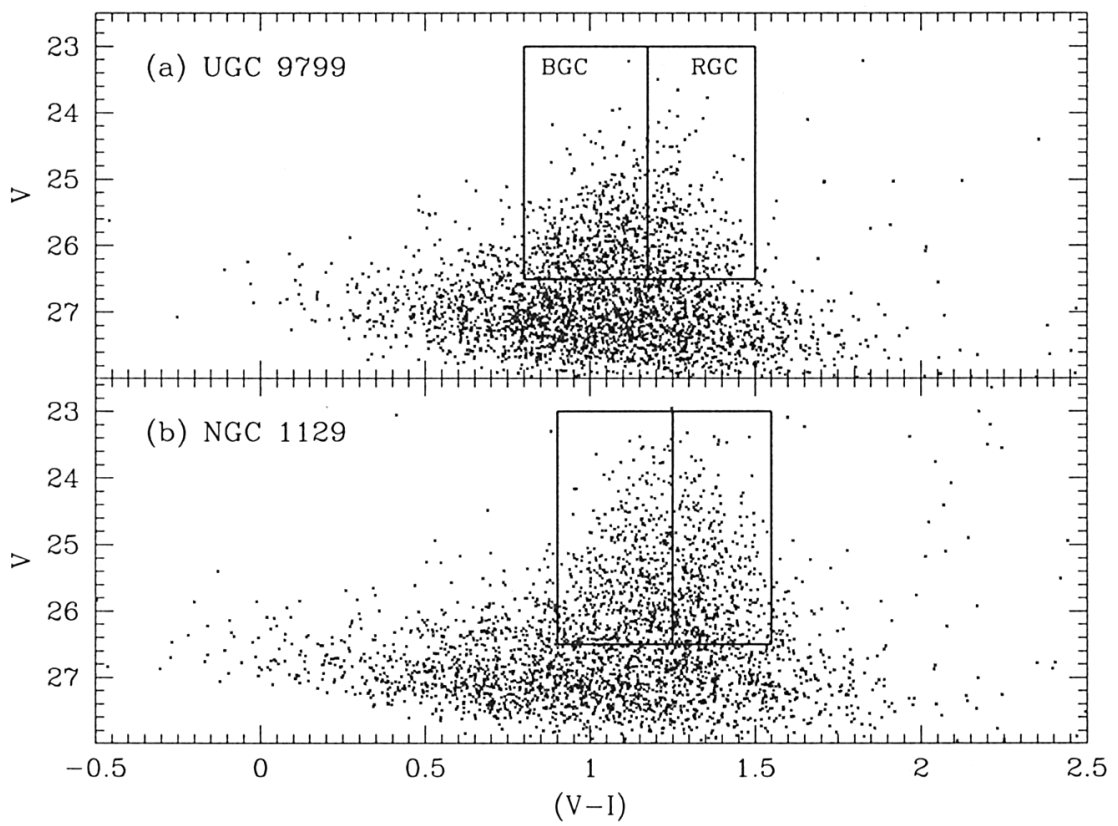

Figure 1. Color-magnitude diagrams of the point sources in UGC 9799 (a) and NGC 1129 (b). The boxes represent the color and magnitude ranges for the bright blue and red globular cluster candidates with $V<26.5$ mag.

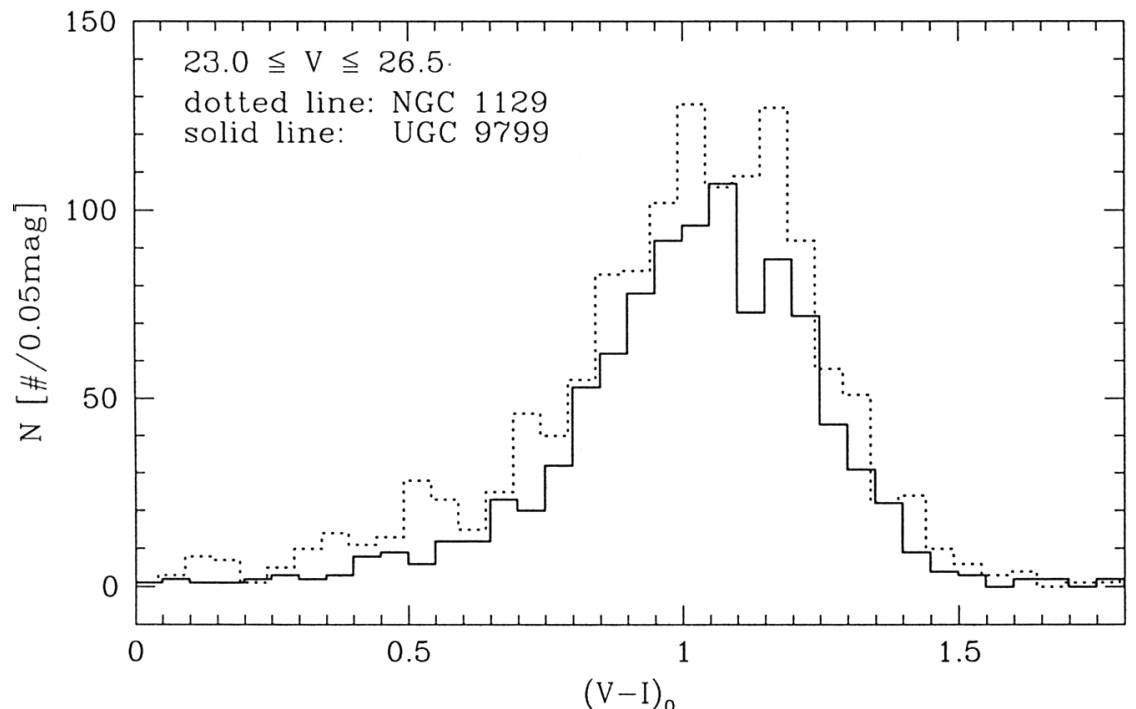

Figure 2. $(V-I)_{0}$ color distributions of the bright point sources (mostly globular clusters) with $V<26.5$ mag in UGC 9799 and NGC 1129 
ular clusters and the color distribution of the globular clusters in both galaxies are similarly bimodal: a blue peak at $(V-I)_{0}=1.07([\mathrm{Fe} / \mathrm{H}]=-0.8)$ and a red peak at $(V-I)_{0}=1.17([\mathrm{Fe} / \mathrm{H}]=-0.4)$ for UGC 9799, and a blue peak at $(V-I)_{0}=1.02([\mathrm{Fe} / \mathrm{H}]=-1.1)$ and a red peak at $(V-I)_{0}=1.17([\mathrm{Fe} / \mathrm{H}]=$ $-0.4)$ for NGC 1129. The number of the bright globular clusters with $V<26.5$ mag we find is 860 for UGC 9799 and 1,060 for NGC 1129. The ratio of the number of the blue globular clusters (BGC) and that of the red globular clusters (RGC) for UGC 9799 is derived to be $\mathrm{N}(\mathrm{BRC}) / \mathrm{N}(\mathrm{RGC})=1.8$, higher than that of NGC 1129, N(BRC)/N(RGC) $=1.3$.

The surface number density profiles of the globular clusters show that the globular clusters in both galaxies are spatially more extended than those of the stellar halo, and the mean colors of the globular clusters are bluer than those of the stellar halo. The RGCs are found to be more centrally concentrated than the RGCs in both galaxies.

Luminosity functions of the globular clusters (GCLFs) are derived after background subtraction and incompleteness correction, but they do not reach the turnovers which are expected to be at $V \approx 28.7 \mathrm{mag}$ for UGC 9799 and $V \approx 27.5 \mathrm{mag}$ for NGC 1129 . We estimate the total number of the globular clusters from the GCLFs, obtaining $\mathrm{N}$ (total)=10,000 \pm 700 for UGC 9799 and $\mathrm{N}($ total $)=7,000 \pm 700$ for NGC 1129. The total number of the globular clusters in UGC 9799 derived in this study is much smaller than that derived from the ground-based observation by Harris et al. (1995).

From the integrated photometry of the galaxies the total magnitudes of the galaxies are estimated to be $V=12.10 \mathrm{mag}$ and $I=10.77 \mathrm{mag}$ for UGC 9799 $\left(r<63^{\prime \prime}\right)$, and $V=10.80 \mathrm{mag}$ and $I=9.41 \mathrm{mag}$ for NGC $1129\left(r<80^{\prime \prime}\right)$. Absolute total magnitudes of the galaxies are derived to be $M_{V}=-24.02 \mathrm{mag}$ and $M_{I}=-25.30 \mathrm{mag}$ for UGC 9799 , and $M_{V}=-24.08 \mathrm{mag}$ and $M_{I}=-25.32$ mag for NGC 1129, showing that both galaxies belong to the brightest galaxies.

Finally we estimate the specific frequency of the globular clusters, $S_{N}=$ $N_{t} \times 10^{0.4\left(M_{V}+15\right)}=2.5 \pm 0.2$ for UGC 9799 and $S_{N}=1.7 \pm 0.2$ for NGC 1129 . These values are significantly lower than those for normal elliptical galaxies, and the value for UGC 9799 is much lower than that based on the ground-based observation (Harris et al. 1995). If we use the total magnitudes of the galaxies given in the literature $\left(M_{V}=-23.4 \mathrm{mag}\right.$ for UGC $9799, M_{V}=-22.88 \mathrm{mag}$ for NGC 1129), we get $S_{N}=4.4 \pm 0.3$ for UGC 9799 and $S_{N}=5.0 \pm 0.5$ for NGC 1129. This result is not consistent with the intracluster globular cluster model that suggests that the globular clusters are not bound to individual galaxies, but bound to the gravitational potential of the clusters (West et al. 1995).

Acknowledgments. This research is supported in part by the MOST/ KISTEP International Collaboration Research Program (1-99-009).

\section{References}

Dolphin, A. E. 2000, PASP, 112, 1383

Harris, W. Pritchet, C. J., \& McClure, R. D. 1995, ApJ, 441, 120

West, M. J., Côte, P., Jones, C., Forman, W., \& Marzke, R. O. 1995, AJ, 453, L77 